\title{
Effets de l'éclaircie précommerciale et de la fertilisation sur un gaulis de 10 ans à dominance de bouleau jaune
}

\author{
par Lise Robitaille ${ }^{1}$, Gilles Sheedy ${ }^{1}$ et Yvon Richard ${ }^{1}$
}

\begin{abstract}
Résumé
Dans un gaulis de l'érablière à bouleau jaune, on a sélectionné un bouleau jaune tous les 4,5 mètres et créé autour de cette tige une colonne de lumière dont le rayon $(\mathrm{R})$ est le même depuis la souche jusqu'au sommet de la cime. Le dispositif comprend trois études et regroupe quatre intensités d'éclaircie $(R=0 \mathrm{~m}$, $\mathrm{R}=0,5 \mathrm{~m}, \mathrm{R}=1,0 \mathrm{~m}, \mathrm{R}=1,5 \mathrm{~m}$ ) et cinq traitements de fertilisation (trois avec N-P-K et deux avec N). Les mesures du d.h.p., de l'étage et de l'élagage naturel portent sur 2258 bouleaux jaunes répartis dans 48 places échantillons; pour la hauteur, 10 pour 100 des tiges ont été mesurées.

Cinq ans plus tard, les résultats des trois études indiquent que l'accroissement en diamètre a augmenté avec l'intensité de l'éclaircie et la dominance des arbres; le dégagement fort $\left(\mathrm{R}_{15}\right)$ donne un accroissement en diamètre de $3,9 \mathrm{~cm}$ pour les dominants, de $3,1 \mathrm{~cm}$ pour les codominants et de $2,6 \mathrm{~cm}$ pour les intermédiaires. Cependant, le dégagement fort défavorise temporairement l'élagage naturel.

La fertilisation a provoqué des augmentations dans les teneurs en $\mathrm{N}$ et $\mathrm{K}$ du feuillage du bouleau jaune, mais n'a eu aucun effet sur la croissance en hauteur et un effet très faible sur la croissance en diamètre dans le cas du dégagement moyen $\left(R_{10}\right)$.
\end{abstract}

Mots clés: Gaulis, éclaircie, dégagement de l'arbre d'avenir, fertilisation, accroissement, élagage naturel, Betula alleghaniensis (Britt.).

\begin{abstract}
One yellow birch was selected every 4.5 metres in a sugar maple-yellow birch sapling stand, and released from competition by removing all stems whose crown extended into a column of equal radius $(\mathrm{R})$ from the stump to the top of the tree. The experiment comprises three studies and includes four thinning intensities $(\mathrm{R}=0 \mathrm{~m}, \mathrm{R}=0,5 \mathrm{~m}, \mathrm{R}=1,0 \mathrm{~m}, \mathrm{R}=1,5 \mathrm{~m})$ and five fertilization treatments (three with N-P-K and two with N). Dbh, story and natural pruning data were taken on 2258 yellow birches distributed in 48 sample plots; heights were measured on 10 per 100 of the trees.

Five years later, the results of the three studies show that dbh growth increased with thinning intensity and tree dominance; for the heavy release $\left(R_{15}\right)$ the dbh increment is $3.9 \mathrm{~cm}$ for the dominants, $3.1 \mathrm{~cm}$ for the codominants and $2.6 \mathrm{~cm}$ for the intermediates. However, heavy release interferes temporarily with natural pruning.

The presence of $\mathrm{N}$ and $\mathrm{K}$ increased in the foliage of yellow birch with fertilization but height increment was not affected and diameter increment was slightly higher with the moderate release $\left(\mathrm{R}_{10}\right)$.
\end{abstract}

Key words: Sapling, thinning, crop-tree release, fertilization, growth, natural pruning, Betula alleghaniensis (Britt.).

traitement n'est pas élevé et seuls des arbres sélection-nés sont dégagés.

Plusieurs études ainsi menées aux États-Unis sur le bouleau jaune ont donné des résultats encourageants (Erdmann et al. 1981, Hannah 1985). D'autres études combinant l'éclaircie et la fertilisation (Ellis 1979, Hannah 1985) ont montré que cette dernière a peu d'importance. Malgré ces résultats, nous avons décidé de faire l'essai au Québec de l'éclaircie et de la fertilisation dans un tel peuplement.

Le but de la présente étude consiste donc à mesurer les effets de différentes intensités d'éclaircie précommerciale, combinées ou non à de la fertilisation, sur la survie, l'accroissement et la qualité (forme) du bouleau jaune.

\section{Aire d'étude et méthodes utilisées}

L'expérience s'est déroulée à la Station forestière de Duchesnay située à $40 \mathrm{~km}$ au nord-ouest de la ville de Québec. Le secteur étudié se trouve à la limite sud de la section forestière Laurentienne (L.4a de Rowe, 1972) dans le domaine de l'érablière à bouleau jaune. Thibault (1985) précise que ce secteur fait partie du sous-domaine de l'érablière à bouleau jaune et hêtre et plus précisément de la région écologique des moyennes Laurentides de la Mauricie.

Le peuplement traité provient d'une coupe rase avec épandage des déchets et débusquage en longueur. Cette coupe eut lieu à l'été 1971 sur une superficie de 58 ha recouverte d'un peuplement feuillu inéquienne qui comportait un volume

'Direction de la recherche et du développement, Ministère de l'Énergie et des Ressources, 2700 rue Einstein, Sainte-Foy, Québec, G1P 3 W8. 
marchand de $189 \mathrm{~m}^{3} /$ ha dont $104 \mathrm{~m}^{3} /$ ha d'érable à sucre, $31 \mathrm{~m}^{3} /$ ha de bouleau jaune et $40 \mathrm{~m}^{3} /$ ha de hêtre; le nombre de tiges marchandes par hectare était de 447 (Robitaille 1971).

Dix ans après la coupe, il y avait 20000 gaules/ha dont 5300 bouleaux jaunes. Nous avons alors décidé de sélectionner environ 500 bouleaux jaunes/ha et de les éclaircir selon différentes intensités afin de mesurer les effets de ce traitement; l'expérience fut complétée par des essais de fertilisation.

Le dispositif expérimental implanté sur une superficie de 17 ha comprend 48 places échantillons de 0,1 ha dans lesquelles en moyenne 50 arbres ont été marqués à la peinture et numérotés à des espacements d'environ 4,5 sur 4,5 m. Ces arbres ont été dégagés et mesurés dans les places traitées et uniquement mesurés dans les places témoins. Ce dispositif comprend les trois études suivantes qui comportent chacune 16 places échantillons.

\section{- Première étude: éclaircie}

Dans la première étude, un bouleau jaune était choisi tous les $4,5 \mathrm{~m}$ et était dégagé en créant autour de lui une colonne de lumière ou colonne de dégagement dont le rayon (R) était mesuré en utilisant l'axe de la tige (tronc) comme centre. Les rayons de dégagement appliqués en juin 1982 sont:

$\mathrm{R}_{15}: \mathrm{R}=1,5 \mathrm{~m}$ pour un dégagement dit fort

$\mathrm{R}_{10}: \mathrm{R}=1,0 \mathrm{~m}$ pour un dégagement dit moyen

$R_{05}: R=0,5 \mathrm{~m}$ pour un dégagement dit léger

$\mathrm{R}_{0}$ : aucun dégagement (témoin).

Le dégagement s'est fait au sécateur qui servait à la fois à mesurer le rayon de dégagement et à couper les compétiteurs à $1 \mathrm{~m}$ du sol pour qu'ils aient moins de chances de retiger.

\section{- Deuxième étude: éclaircie et un traitement de fertilisation} $\left(F_{l}\right)$

Dans la deuxième étude, les arbres ont été dégagés comme dans la première et ont été ensuite fertilisés. Le traitement de fertilisation $\left(\mathrm{F}_{1}\right)$ consiste en l'application de $150 \mathrm{~kg} / \mathrm{ha}$ d'azote sous forme d'urée ( 45 p. $100 \mathrm{de} \mathrm{N}), 75 \mathrm{~kg} / \mathrm{ha}$ de phosphore sous forme de triple superphosphate $(45$ p. 100 de $\mathrm{P}_{2} \mathrm{O}_{5}$ ) et $75 \mathrm{~kg} / \mathrm{ha}$ de potassium sous forme de chlorure de potassium $\left(60\right.$ p. 100 de $\left.\mathrm{K}_{2} \mathrm{O}\right)$. L'engrais a été appliqué par pied d'arbre en juillet 1982 sur un rayon de $1 \mathrm{~m}$ autour des arbres dégagés.

- Troisième étude: éclaircie $\left(R_{10}\right)$ et quatre traitements de fertilisation $\left(F_{2}, F_{3}, F_{4}\right.$ et $\left.F_{5}\right)$

La troisième étude a retenu un seul rayon de dégagement, celui de $1 \mathrm{~m}\left(\mathrm{R}_{10}\right)$, et quatre traitements de fertilisation, soit:

$F_{2}: 250 \mathrm{~kg} / \mathrm{ha}$ de $\mathrm{N}, 75 \mathrm{~kg} / \mathrm{ha}$ de $\mathrm{P}$ et $75 \mathrm{~kg} / \mathrm{ha}$ de $\mathrm{K}$ $F_{3}: 75 \mathrm{~kg} / \mathrm{ha}$ de $\mathrm{N}, 75 \mathrm{~kg} / \mathrm{ha}$ de $\mathrm{P}$ et $75 \mathrm{~kg} / \mathrm{ha}$ de $\mathrm{K}$

$\mathrm{F}_{4}: 150 \mathrm{~kg} / \mathrm{ha}$ de N

$\mathrm{F}_{5}: 250 \mathrm{~kg} / \mathrm{ha}$ de $\mathrm{N}$.

Les engrais sont sous la même forme que dans la deuxième étude et ils ont été répartis de la même façon.

\section{- Mesures prises}

Dans chacune des 48 places échantillons, on a pris les mesures suivantes:
- pour les 50 arbres numérotés: l'état de l'arbre, l'étage et le diamètre à hauteur de poitrine (d.h.p.) au moment du traitement puis 5 ans plus tard. On a aussi mesuré pour ces mêmes arbres le diamètre de la plus grosse branche le long du tronc dans les premiers $2,4 \mathrm{~m}$ ou première bille, 5 ans après le traitement, afin d'évaluer le degré d'élagage naturel;

- pour 5 arbres de l'étage dominant ou codominant, la hauteur totale au moment du traitement puis 5 ans plus tard.

Pour déterminer l'étage, on a utilisé la classification du Service de l'inventaire forestier du ministère de l'Énergie et des Ressources (Noël et Rouleau 1979):

"Dominant se dit de tout arbre dont la hauteur dépasse le niveau moyen de l'ensemble du peuplement et dont la cime et une partie de la couronne reçoivent pleine lumière de tous les côtés. Il est ordinairement plus gros que l'arbre moyen du peuplement.

Codominant se dit de tout arbre dont la hauteur ne dépasse pas la hauteur moyenne de l'ensemble du peuplement et dont seule la cime reçoit directement la lumière.

Intermédiaire se dit de tout arbre dont la hauteur est inférieure à la hauteur moyenne du peuplement et dont la cime ne reçoit que très peu de lumière directe; c'est ordinairement un sujet retardataire.

Supprimé se dit de tout arbre dont la cime est entièrement en-dessous du niveau général de la voûte foliacée et complètement privée de lumière directe." On a dégagé par erreur quelques arbres supprimés, mais on n'en a pas tenu compte dans l'étude.

Enfin, à l'été 1982, 144 échantillons foliaires ont été prélevés sur trois arbres par place d'étude, au début de septembre de la même année. L'analyse chimique de ces échantillons a été réalisée au laboratoire selon les méthodes usuelles (Walsh 1971).

\section{- Analyse des données}

À cause des particularités du dispositif expérimental, il n'a pas été possible de regrouper les études lors de l'analyse des données. Pour chaque étude, on a procédé à une analyse de la covariance pour un plan factoriel à deux voies en ajoutant au facteur déjà décrit l'étage des arbres dégagés. La covariable était le diamètre avant traitement.

Les analyses ont été effectuées pour deux variables soit le diamètre à hauteur de poitrine et la hauteur totale.

\section{Résultats}

\section{A) Effet du dégagement et de la fertilisation sur les concen- trations en éléments nutritifs du feuillage du bouleau jaune}

Les résultats des analyses foliaires présentés au tableau 1 montrent bien que la fertilisation a eu des effets positifs sur les teneurs en azote $(\mathrm{N})$ et en potassium $(\mathrm{K})$ du bouleau jaune. Ainsi, la teneur en $\mathrm{N}$ des feuilles provenant des places témoins $\left(\mathrm{F}_{0} \mathrm{R}_{0}\right)$ est de 1,93 p. 100 , alors qu'elle est de 2,25 p. 100 pour le traitement $F_{1} R_{0}$; il en va de même pour la teneur en $\mathrm{K}$ qui passe de 0,68 à 0,84 p. 100 pour les mêmes traitements. Le dégagement a aussi un effet positif sur les concentrations en éléments du feuillage. Dans l'ensemble, les concentrations en éléments nutritifs des feuilles semblent suffisantes pour assurer une bonne croissance des arbres. 
Tableau 1. Résultats de l'analyse foliaire de 1982 à la suite du dégagement et de la fertilisation.

Concentrations en éléments nutritifs dans les feuilles en p. 100 (moyenne pour 12 échantillons par traitement)

\begin{tabular}{lccccc}
\cline { 2 - 6 } Traitement & $\mathbf{N}$ & $\mathbf{P}$ & $\mathbf{K}$ & $\mathbf{M g}$ & $\mathbf{C a}$ \\
\hline $\mathrm{F}_{0} \mathrm{R}_{0}$ & 1,93 & 0,22 & 0,68 & 0,24 & 0,85 \\
$\mathrm{~F}_{1} \mathrm{R}_{0}$ & 2,25 & 0,23 & 0,84 & 0,21 & 0,90 \\
Dégagé $^{1}$ & 2,01 & 0,25 & 0,70 & 0,24 & 0,85 \\
Déggagé et fertilisé $^{2}$ & 2,09 & 0,30 & 0,81 & 0,24 & 0,87 \\
$\mathrm{~F}_{0} \mathrm{R}_{10}$ & 1,84 & 0,23 & 0,68 & 0,25 & 0,77 \\
$\mathrm{~F}_{1} \mathrm{R}_{10}$ & 2,20 & 0,27 & 0,67 & 0,25 & 0,89 \\
$\mathrm{~F}_{2} \mathrm{R}_{10}$ & 2,23 & 0,27 & 0,83 & 0,21 & 0,72 \\
$\mathrm{~F}_{3} \mathrm{R}_{10}$ & 1,87 & 0,30 & 0,88 & 0,20 & 0,83 \\
$\mathrm{~F}_{4} \mathrm{R}_{10}$ & 1,91 & 0,17 & 0,74 & 0,21 & 0,88 \\
$\mathrm{~F}_{5} \mathrm{R}_{10}$ & 2,06 & 0,17 & 0,78 & 0,22 & 0,87 \\
\hline
\end{tabular}

${ }^{1}$ Moyenne des traitements $F_{0} R_{05}, F_{0} R_{10}$ et $F_{0} R_{15}$ pour 36 échantillons.

${ }^{2}$ Moyenne des traitements $F_{1} R_{05}, F_{1} R_{10}$ et $F_{1} R_{15}$ pour 36 échantillons.

\section{B) Accroissement en diamètre du bouleau jaune}

Les principaux résultats apparaissent au tableau $2 . \mathrm{La}$ première étude montre qu'entre 10 et 15 ans, l'accroissement moyen en diamètre du bouleau jaune augmente avec l'intensité du dégagement. Si l'on prend la moyenne de toutes les tiges dégagées, il est de $1,5 \mathrm{~cm}$ pour $\mathrm{R}_{0} \mathrm{~F}_{0}$, de $2,1 \mathrm{~cm}$ pour $\mathrm{R}_{05} \mathrm{~F}_{0}$, de $2,5 \mathrm{~cm}$ pour $\mathrm{R}_{10} \mathrm{~F}_{0}$ et de $3,2 \mathrm{~cm}$ pour $\mathrm{R}_{15} \mathrm{~F}_{0}$.

Selon l'étage que les arbres occupaient au moment du traitement à l'âge de 10 ans, l'effet du dégagement se fait sentir par ordre décroissant chez les dominants, puis chez les codominants et enfin chez les intermédiaires. Chez les dominants, l'accroissement est de $2,6 \mathrm{~cm}$ pour $\mathrm{R}_{0} \mathrm{~F}_{0}$, de $3,2 \mathrm{~cm}$ pour $\mathrm{R}_{05} \mathrm{~F}_{0}$ et $\mathrm{R}_{10} \mathrm{~F}_{0}$ et de $3,9 \mathrm{~cm}$ pour $\mathrm{R}_{15} \mathrm{~F}_{0}$; chez les codominants, il est de $1,6 \mathrm{~cm}$ pour $\mathrm{R}_{0} \mathrm{~F}_{0}$, de $2,0 \mathrm{~cm}$ pour $\mathrm{R}_{05} \mathrm{~F}_{0}$, de $2,5 \mathrm{~cm}$ pour $\mathrm{R}_{10} \mathrm{~F}_{0}$ et de $3,1 \mathrm{~cm}$ pour $\mathrm{R}_{15} \mathrm{~F}_{0}$; enfin, chez les intermédiaires l'accroissement est de $1,1 \mathrm{~cm}$ pour $\mathrm{R}_{0} \mathrm{~F}_{0}$, de $1,3 \mathrm{~cm}$ pour $\mathrm{R}_{05} \mathrm{~F}_{0}$, de $2,0 \mathrm{~cm}$ pour $\mathrm{R}_{10} \mathrm{~F}_{0}$ et de $2,6 \mathrm{~cm}$ pour $\mathrm{R}_{15} \mathrm{~F}_{0}$.

Dans cette première étude, le tableau d'analyse de la covariance (tableau 3) indique des différences très significatives entre les étages et les rayons de dégagement. Par contre, l'interaction entre les deux facteurs n'est pas significative. Les graphiques (figure 1) illustrent bien ces différences. Plus l'étage est élevé, plus l'accroissement est élevé; de même, plus le rayon de dégagement est grand, plus l'accroissement est élevé. Dans ce dernier cas l'analyse montre que les tendances linéaire, quadratique et cubique sont significatives. Le graphique montre bien les changements de pente entre les divers rayons, avec la partie la plus abrupte entre $R_{10}$ et $R_{15}$.

Le tableau 4 présente les valeurs ajustées selon les combinaisons des facteurs ainsi que les gains de chaque combinaison par rapport au témoin $\mathrm{R}_{0}$. Ce dernier point fait ressortir que les dominants présentent les gains les plus faibles sauf à $\mathrm{R}_{05}$ tandis que ce sont les intermédiaires et les codominants qui profitent le plus du dégagement à $R_{10}$ et $\mathrm{R}_{15}$.

Dans la deuxième étude, l'interaction entre les étages et les rayons de dégagement étant significative (tableau 3), on ne peut analyser séparément chaque facteur même s'ils sont eux aussi différents significativement. Les valeurs par combinaison des facteurs sont présentées au tableau 4 et à la figure 2 qui illustre bien l'interaction. $\grave{A} R_{0}$ et $R_{05}$,

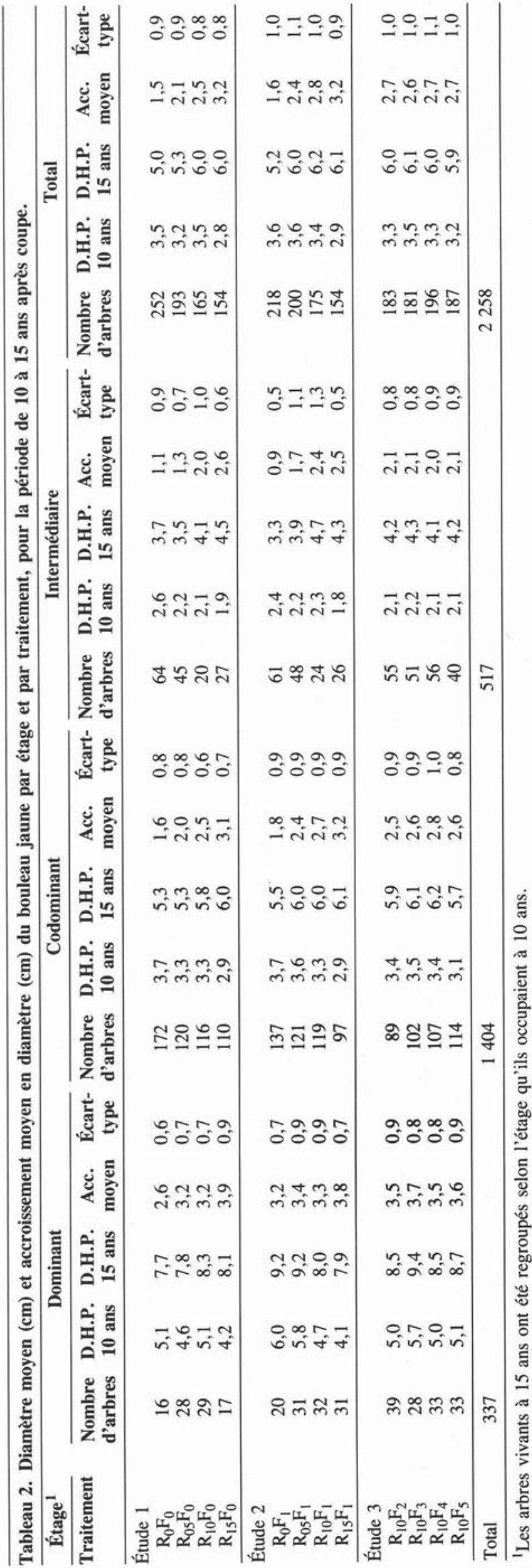


Tableau 3. Analyse de la covariance: accroissement en diamètre selon quatre rayons de dégagement, sans fertilisation (étude 1) ou avec fertilisation (étude 2).

\begin{tabular}{|c|c|c|c|c|c|c|}
\hline \multirow[b]{2}{*}{$\begin{array}{l}\text { Source de la } \\
\text { variation }\end{array}$} & \multicolumn{3}{|c|}{ Étude 1: non fertilisé } & \multicolumn{3}{|c|}{ Étude 2: fertilisé avec $F_{1}$} \\
\hline & $\begin{array}{c}\text { Degrés de } \\
\text { liberté }\end{array}$ & $\mathbf{F}$ & $\mathbf{P}$ & $\begin{array}{c}\text { Degrés de } \\
\text { liberté }\end{array}$ & $\mathbf{F}$ & $\mathbf{P}$ \\
\hline Étage & 2 & 95,47 & 0,0001 & 2 & 65,25 & 0,0001 \\
\hline Rayon de dégagement & 3 & 131,40 & 0,0001 & 3 & 108,51 & 0,0001 \\
\hline Effet linéaire & 1 & 453,63 & 0,0001 & 1 & 324,89 & 0,0001 \\
\hline Effet quadratique & 1 & 5,49 & 0,0194 & 1 & 0,58 & 0,4449 \\
\hline Effet cubique & 1 & 5,89 & 0,0154 & 1 & 0,07 & 0,7936 \\
\hline Étage $\times$ rayon & 6 & 1,68 & 0,1224 & 6 & 2,04 & $>0,0500$ \\
\hline Covariable & 1 & & & 1 & & \\
\hline Erreur & 751 & & & 733 & & \\
\hline
\end{tabular}

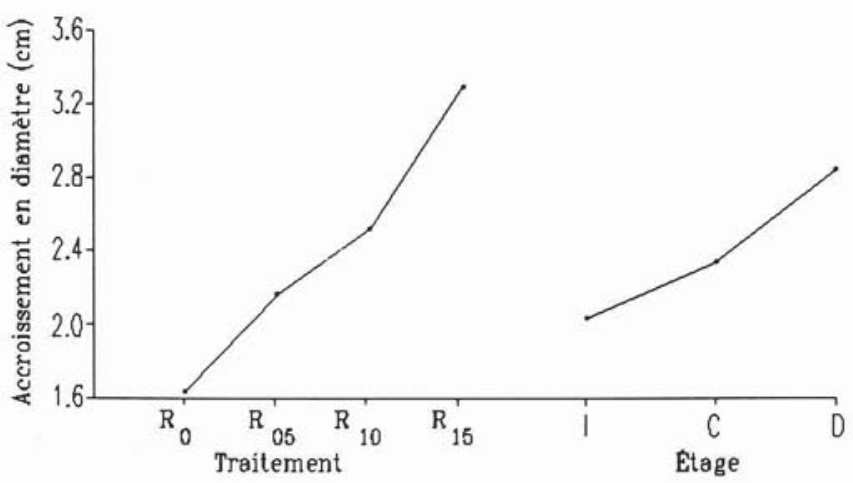

Figure 1. Accroissement quinquennal en diamètre (valeurs ajustées) par rayon de dégagement et par étage. Étude 1: non fertilisé. D: dominant. C: codominant. $\mathrm{I}$ : intermédiaire. $\mathrm{R}_{0}$ : aucun dégagement. $\mathrm{R}_{05}$ : dégagement $50 \mathrm{~cm}$. $\mathrm{R}_{10}$ : dégagement $1,0 \mathrm{~m}$. $\mathrm{R}_{15}$ : dégagement $1,5 \mathrm{~m}$.

l'accroissement en diamètre est supérieur chez les dominants et faible chez les intermédiaires; il se situe entre eux chez les codominants. À $\mathrm{R}_{10}$, l'accroissement est le même pour les trois étages alors qu'à $\mathrm{R}_{15}$, les dominants et les codominants sont égaux et légèrement supérieurs aux intermédiaires.

Lorsqu'on examine les gains par rapport à $\mathrm{R}_{0}$ (tableau 4), on remarque que les dominants gagnent plus que les autres à $\mathrm{R}_{05}$; comme dans l'étude 1 , ils profitent mieux que les autres d'un dégagement léger. Par contre, ce sont les intermédiaires et les codominants qui profitent le plus des dégagements $R_{10}$ et $R_{15}$ tout comme dans l'étude 1 mais de façon un peu plus marquée cependant.

Le dispositif expérimental de la troisième étude en est un à blocs complets. L'analyse de la covariance (tableau 5) donne des différences très significatives entre les répétitions et entre les étages mais non entre les traitements de fertilisation; de plus, l'interaction entre les répétitions et la fertilisation est significative, ce qui indique des réactions différentes à la fertilisation selon les répétitions. L'interprétation de cette interaction n'est pas effectuée parce qu'aucun critère pouvant laisser supposer une telle interaction n'a été retenu lors de la mise en place du dispositif.

Il existe donc une différence très significative entre les étages. Comme l'indiquent les moyennes ajustées, plus l'étage est élevé, plus l'accroissement en diamètre est élevé; il est de $3,1 \mathrm{~cm}$ chez les dominants, de $2,7 \mathrm{~cm}$ chez les codominants et de $2,4 \mathrm{~cm}$ chez les intermédiaires.
Par contre, il n'y a pas de différences d'accroissement en diamètre entre les traitements de fertilisation, comme l'indiquent les moyennes: soit $2,7 \mathrm{~cm}$ pour $\mathrm{F}_{2}$ et $\mathrm{F}_{4}, 2,6 \mathrm{~cm}$ pour $\mathrm{F}_{3}$ et $2,8 \mathrm{~cm}$ pour $\mathrm{F}_{5}$. Enfin, si on compare le meilleur traitement de fertilisation de cette étude, $\mathrm{R}_{10} \mathrm{~F}_{5}$, avec le traitement $\mathrm{R}_{10} \mathrm{~F}_{0}$, qui ont respectivement un accroissement en diamètre de 2,8 et $2,5 \mathrm{~cm}$, la différence est trop faible pour qu'on puisse conclure que la fertilisation a un effet suffisamment important pour en justifier l'application.

\section{C) Accroissement en hauteur du bouleau jaune}

La hauteur a été mesurée sur 10 p. 100 des tiges dégagées soit 234 bouleaux jaunes dominants et codominants. Les résultats regroupés au tableau 6 montrent que l'étage ou la fertilisation n'ont pas d'effets sur l'accroissement en hauteur. Ces conclusions ont été confirmées par des analyses de la covariance dont les résultats ne sont pas présentés parce qu'ils ne démontrent aucune différence significative ou quasi telle.

Ces résultats étaient prévisibles puisque l'on reconnaît que l'accroissement en hauteur est peu affecté par la densité sauf par les densités extrêmes. Dans ces études, aucun des dégagements ne modifie la densité d'une façon assez marquée pour affecter l'accroissement en hauteur. Il ne fallait donc pas s'attendre à des différences importantes.

\section{D) Élagage naturel}

Le développement des branches le long du tronc dans la première bille a été observé sur toutes les tiges dégagées. Les résultats montrent que la fertilisation n'a eu aucun effet sur ce développement; ceci peut s'expliquer par le fait que les dégagements effectués, même le plus fort $\left(R_{15}\right)$, étaient trop légers et par la présence des arbres avoisinants qui ont aussi bénéficié des engrais.

Les résultats des trois études ont donc été regroupés en un seul tableau en ne considérant que le rayon de dégagement. Ces résultats sont tellement évidents qu'ils ne justifient pas une analyse statistique. On s'aperçoit qu'entre 10 et 15 ans, l'élagage se fait beaucoup moins bien à $R_{15}$ puisque seulement 8 p. 100 des bouleaux jaunes ne comportent aucune branche dans la première bille contre 43 p. 100 à $R_{10}, 50$ p. 100 à $R_{05}$ et 58 p. 100 à $R_{0}$ (tableau 7). Inversement, la proportion de branches de 1 à $3 \mathrm{~cm}$ de diamètre le long du tronc augmente avec l'intensité du dégagement.

Ces résultats montrent que si on utilise $R_{15}$ pour obtenir un meilleur accroissement en diamètre, on pourrait s'attendre à devoir élaguer les arbres par la suite. Quant aux plus 
Tableau 4. Accroissement moyen en diamètre (valeurs ajustées par étage et par rayon de dégagement et différence par rapport au témoin.

\begin{tabular}{|c|c|c|c|c|c|c|c|c|}
\hline \multirow[b]{2}{*}{ Étage } & \multicolumn{4}{|c|}{ Étude 1: non fertilisé } & \multicolumn{4}{|c|}{ Étude 2: fertilisé avec $F_{1}$} \\
\hline & $\begin{array}{c}\text { Dominant } \\
\mathrm{cm}\end{array}$ & $\begin{array}{c}\text { Codominant } \\
\mathrm{cm}\end{array}$ & $\begin{array}{c}\text { Intermédiaire } \\
\text { cm }\end{array}$ & $\begin{array}{c}\text { Total } \\
\text { cm }\end{array}$ & $\begin{array}{c}\text { Dominant } \\
\mathrm{cm}\end{array}$ & $\begin{array}{c}\text { Codominant } \\
\mathrm{cm}\end{array}$ & $\begin{array}{c}\text { Intermédiaire } \\
\mathrm{cm}\end{array}$ & $\begin{array}{c}\text { Total } \\
\mathrm{cm}\end{array}$ \\
\hline \multicolumn{9}{|l|}{ Traitement } \\
\hline $\mathrm{R}_{0}$ & 2,11 & 1,51 & 1,29 & 1,64 & 1,93 & 1,61 & 1,01 & 1,52 \\
\hline $\mathrm{R}_{05}$ & 2,83 & 2,08 & 1,58 & 2,16 & 3,34 & 2,26 & 0,73 & 2,11 \\
\hline $\mathrm{R}_{10}$ & 2,78 & 2,45 & 2,32 & 2,52 & 2,99 & 2,82 & 2,92 & 2,91 \\
\hline $\mathrm{R}_{15}$ & 3,64 & 3,27 & 2,98 & 3,30 & 3,47 & 3,50 & 2,99 & 3,32 \\
\hline Total & 2,84 & 2,33 & 2,04 & & 2,94 & 2,55 & 1,91 & \\
\hline \multicolumn{9}{|l|}{ Différence } \\
\hline $\mathrm{R}_{05}-\mathrm{R}_{0}$ & 0,72 & 0,57 & 0,29 & & 1,41 & 0,65 & $-0,28$ & \\
\hline $\mathrm{R}_{10}-\mathrm{R}_{0}$ & 0,67 & 0,94 & 1,03 & & 1,06 & 1,21 & 1,91 & \\
\hline $\mathrm{R}_{15}-\mathrm{R}_{0}$ & 1,53 & 1,76 & 1,69 & & 1,54 & 1,89 & 1,98 & \\
\hline
\end{tabular}

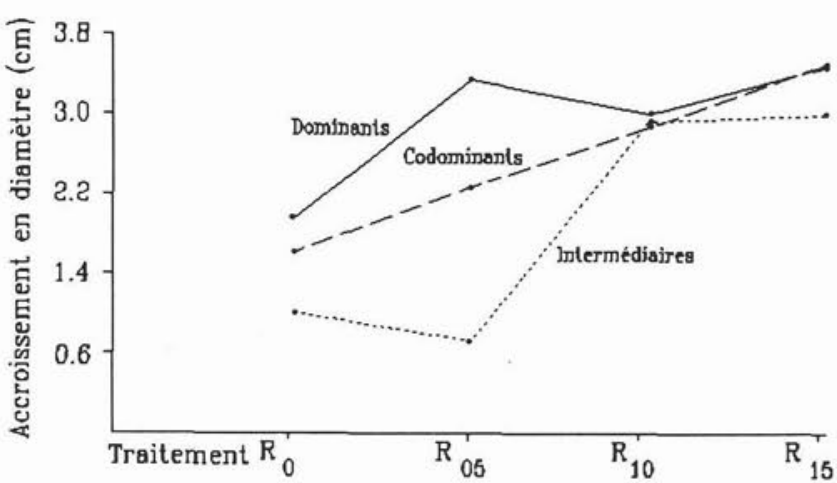

Figure 2. Accroissement quinquennal en diamètre (valeurs ajustées): interaction entre les étages et les rayons de dégagement. Étude 2: fertilisé avec $\mathrm{F}_{1}(150 \mathrm{~N}-75 \mathrm{P}-75 \mathrm{~K})$.

grosses branches dont le diamètre varie entre 4 et $9 \mathrm{~cm}$, on les retrouve sur près de 2 p. 100 des arbres dans toutes les catégories de dégagement; ces grosses branches devaient être présentes lors du dégagement et on aurait alors eu intérêt à les élaguer.

\section{Discussion et conclusion}

Dans l'ensemble, les résultats montrent que l'éclaircie précommerciale au stade du gaulis a eu des effets marqués, surtout sur la croissance en diamètre du bouleau jaune.

Les résultats des trois études concordent quant aux différences entre les tiges, à savoir: plus l'étage est élevé, plus l'accroissement en diamètre est élevé. Par contre, les codominants et les intermédiaires profitent plus que les dominants du dégagement sur cette période de cinq ans. Nous recommanderions donc de dégager les codominants et les dominants car il n'est pas certain que les intermédiaires qui bénéficient d'un puits de lumière temporaire parviendront à devenir codominants et ainsi à se retrouver dans le couvert principal et à bien continuer à croître. Lamson et Smith (1987) suggèrent d'ailleurs de choisir les tiges d'avenir parmi les dominants et codominants de grande qualité et ayant des cimes vigoureuses.

Quant aux différences entre les rayons de dégagement, les deux premières études concordent et indiquent que plus le rayon est grand plus l'accroissement est élevé. On est donc porté à recommander le plus grand rayon de dégagement soit
Tableau 5. Analyse de la covariance: accroissement en diamètre. Étude 3: $R_{10}$ et fertilisé avec $F_{2}, F_{3}, F_{4}$ et $F_{5}$.

\begin{tabular}{lccc}
\hline & \multicolumn{3}{c}{ Accroissement en diamètre } \\
\cline { 2 - 4 } $\begin{array}{l}\text { Source de la } \\
\text { variation }\end{array}$ & $\begin{array}{l}\text { Degrés de } \\
\text { liberté }\end{array}$ & $\mathbf{F}$ & $\mathbf{P}$ \\
\hline Répétitions & 3 & 31,14 & 0,0001 \\
Étage & 2 & 11,89 & 0,0001 \\
Fertilisation & 3 & 1,04 & 0,3723 \\
Répétitions & 9 & 3,04 & 0,0014 \\
fertilisation & 1 & & \\
Covariable & 728 & & \\
Erreur & & & \\
\hline
\end{tabular}

1,5 m. Cependant, ce rayon de 1,5 m défavorise l'élagage naturel au cours des premiers cinq ans suivant le traitement. Toutefois, lors d'une vérification oculaire sept ans après le traitement, on a constaté que plusieurs branches le long du tronc avaient séché; le couvert forestier s'est refermé et les arbres qui entourent la colonne de dégagement continuent eux aussi à s'accroître en hauteur et à compétitionner avec les arbres dégagés. Il ne faut pas oublier qu'au moment du traitement à l'âge de 10 ans, la hauteur moyenne des bouleaux jaunes dominants et codominants n'était que de $5,6 \mathrm{~m}$, soit environ le quart de la hauteur que cette espèce atteint à maturité à Duchesnay. Lors du remesurage de 10 ans en 1992, on pourra vraiment savoir si le rayon de $1,5 \mathrm{~m}$ constitue dans le présent cas le meilleur traitement. D'ailleurs, ce rayon de $1,5 \mathrm{~m}$ est quand même conservateur car Erdmann et al. (1981) ont trouvé, pour un peuplement d'érable à sucre et de bouleau jaune éclairci à l'âge de sept ans, que "le meilleur compromis entre le taux de croissance et la qualité des tiges appartient, sans autre intervention, à un dépressage qui assure autour de l'arbre d'avenir un rayon de $2,4 \mathrm{~m}$ ". Au delà de ce rayon les tiges fourchent et prennent l'aspect d'arbres fruitiers.

Pour ce qui est des effets de la fertilisation sur l'accroissement en diamètre, le dispositif ne permet malheureusement pas d'en faire une analyse globale et les résultats des différentes études sont trop partiels pour arriver à une recommandation quant à la fertilisation. Cependant, les effets de la fertilisation se sont manifestés au niveau du feuillage où l'on a observé des augmentations dans les teneurs en azote $(\mathrm{N})$ et en potassium (K).

Ces résultats sont d'ailleurs conformes à ceux d'Ellis (1979) et de Hannah (1985). Selon ces auteurs, la fertilisation 
Tableau 6. Hauteur moyenne à 10 et à 15 ans après coupe et accroissement moyen en hauteur pour les arbres de l'étage dominant et de l'étage codominant.

\begin{tabular}{|c|c|c|c|c|c|c|c|c|c|c|c|c|c|c|c|}
\hline \multirow{2}{*}{$\frac{\text { Étage }^{1}}{\text { Traitement }^{1}}$} & \multicolumn{5}{|c|}{ Dominant } & \multicolumn{5}{|c|}{ Codominant } & \multicolumn{5}{|c|}{ Total } \\
\hline & $\begin{array}{l}\text { Nombre } \\
\text { d'arbres }\end{array}$ & $\begin{array}{c}10 \text { ans } \\
\mathrm{m}\end{array}$ & $\begin{array}{c}15 \text { ans } \\
\mathrm{m}\end{array}$ & $\begin{array}{c}\text { Acc. } \\
\text { m }\end{array}$ & $\begin{array}{c}\text { Écart- } \\
\text { type } \\
\text { m }\end{array}$ & $\begin{array}{l}\text { Nombre } \\
\text { d'arbres }\end{array}$ & $\begin{array}{c}10 \text { ans } \\
\mathrm{m}\end{array}$ & $\begin{array}{c}15 \text { ans } \\
\mathrm{m}\end{array}$ & $\begin{array}{c}\text { Acc. } \\
\text { m }\end{array}$ & $\begin{array}{c}\text { Écart- } \\
\text { type } \\
\text { m }\end{array}$ & $\begin{array}{l}\text { Nombre } \\
\text { d'arbres }\end{array}$ & $\begin{array}{c}10 \text { ans } \\
\mathrm{m}\end{array}$ & $\begin{array}{c}15 \text { ans } \\
\mathrm{m}\end{array}$ & $\begin{array}{c}\text { Acc. } \\
\text { m }\end{array}$ & $\begin{array}{c}\text { Ecart- } \\
\text { type } \\
\text { m }\end{array}$ \\
\hline $\begin{array}{l}\text { Étude } 1 \\
\mathrm{R}_{0} \mathrm{~F}_{0} \\
\mathrm{R}_{05} \mathrm{~F}_{0} \\
\mathrm{R}_{10} \mathrm{~F}_{0} \\
\mathrm{R}_{15} \mathrm{~F}_{0} \\
\end{array}$ & $\begin{array}{r}6 \\
10 \\
7 \\
9 \\
\end{array}$ & $\begin{array}{l}6,57 \\
5,85 \\
5,88 \\
5,59 \\
\end{array}$ & $\begin{array}{l}8,80 \\
8,58 \\
8,19 \\
7,94 \\
\end{array}$ & $\begin{array}{l}2,23 \\
2,73 \\
2,31 \\
2,35 \\
\end{array}$ & $\begin{array}{l}0,32 \\
0,51 \\
0,78 \\
0,31 \\
\end{array}$ & $\begin{array}{r}13 \\
9 \\
13 \\
11 \\
\end{array}$ & $\begin{array}{l}6,09 \\
5,57 \\
5,03 \\
4,99 \\
\end{array}$ & $\begin{array}{l}8,69 \\
7,91 \\
7,21 \\
7,32 \\
\end{array}$ & $\begin{array}{l}2,60 \\
2,34 \\
2,18 \\
2,33 \\
\end{array}$ & $\begin{array}{l}0,56 \\
0,33 \\
0,29 \\
0,36 \\
\end{array}$ & $\begin{array}{l}19 \\
19 \\
20 \\
20 \\
\end{array}$ & $\begin{array}{l}6,24 \\
5,72 \\
5,33 \\
5,26 \\
\end{array}$ & $\begin{array}{l}8,72 \\
8,26 \\
7,56 \\
7,60 \\
\end{array}$ & $\begin{array}{l}2,48 \\
2,54 \\
2,23 \\
2,34 \\
\end{array}$ & $\begin{array}{l}0,52 \\
0,47 \\
0,50 \\
0,33 \\
\end{array}$ \\
\hline $\begin{array}{l}\text { Étude } 2 \\
\mathrm{R}_{0} \mathrm{~F}_{1} \\
\mathrm{R}_{05} \mathrm{~F}_{1} \\
\mathrm{R}_{10} \mathrm{~F}_{1} \\
\mathrm{R}_{15} \mathrm{~F}_{1} \\
\end{array}$ & $\begin{array}{l}4 \\
5 \\
6 \\
5 \\
\end{array}$ & $\begin{array}{l}7,04 \\
6,82 \\
5,73 \\
5,32 \\
\end{array}$ & $\begin{array}{l}9,28 \\
9,35 \\
8,45 \\
7,45 \\
\end{array}$ & $\begin{array}{l}2,24 \\
2,53 \\
2,72 \\
2,13 \\
\end{array}$ & $\begin{array}{l}0,77 \\
0,54 \\
0,30 \\
0,59\end{array}$ & $\begin{array}{l}16 \\
15 \\
14 \\
15 \\
\end{array}$ & $\begin{array}{l}6,02 \\
5,59 \\
5,17 \\
5,08 \\
\end{array}$ & $\begin{array}{l}8,34 \\
8,06 \\
7,53 \\
7,74 \\
\end{array}$ & $\begin{array}{l}2,32 \\
2,47 \\
2,36 \\
2,66 \\
\end{array}$ & $\begin{array}{l}0,75 \\
0,60 \\
0,66 \\
0,39 \\
\end{array}$ & $\begin{array}{l}20 \\
20 \\
20 \\
20\end{array}$ & $\begin{array}{l}6,23 \\
5,90 \\
5,34 \\
5,14\end{array}$ & $\begin{array}{l}8,53 \\
8,38 \\
7,81 \\
7,67 \\
\end{array}$ & $\begin{array}{l}2,30 \\
2,48 \\
2,47 \\
2,53 \\
\end{array}$ & $\begin{array}{l}0,73 \\
0,57 \\
0,59 \\
0,49 \\
\end{array}$ \\
\hline $\begin{array}{l}\text { Étude } 3 \\
\mathrm{R}_{10} \mathrm{~F}_{2} \\
\mathrm{R}_{10} \mathrm{~F}_{3} \\
\mathrm{R}_{10} \mathrm{~F}_{4} \\
\mathrm{R}_{10} \mathrm{~F}_{5} \\
\end{array}$ & $\begin{array}{l}7 \\
9 \\
7 \\
7 \\
\end{array}$ & $\begin{array}{l}6,15 \\
6,39 \\
5,63 \\
6,18 \\
\end{array}$ & $\begin{array}{l}9,10 \\
8,88 \\
8,09 \\
8,73 \\
\end{array}$ & $\begin{array}{l}2,95 \\
2,49 \\
2,46 \\
2,55 \\
\end{array}$ & $\begin{array}{l}0,34 \\
0,49 \\
0,53 \\
0,52\end{array}$ & $\begin{array}{l}10 \\
11 \\
12 \\
13\end{array}$ & $\begin{array}{l}4,88 \\
5,54 \\
5,08 \\
5,21 \\
\end{array}$ & $\begin{array}{l}7,62 \\
8,33 \\
7,99 \\
7,98 \\
\end{array}$ & $\begin{array}{l}2,74 \\
2,79 \\
2,91 \\
2,77 \\
\end{array}$ & $\begin{array}{l}0,45 \\
0,43 \\
0,42 \\
0,66\end{array}$ & $\begin{array}{l}17 \\
20 \\
19 \\
20 \\
\end{array}$ & $\begin{array}{l}5,40 \\
5,92 \\
5,28 \\
5,55\end{array}$ & $\begin{array}{l}8,23 \\
8,58 \\
8,02 \\
8,24 \\
\end{array}$ & $\begin{array}{l}2,83 \\
2,66 \\
2,74 \\
2,69 \\
\end{array}$ & $\begin{array}{l}0,41 \\
0,48 \\
0,50 \\
0,61 \\
\end{array}$ \\
\hline Total & 82 & & & & & 152 & & & & & 234 & & & & \\
\hline
\end{tabular}

${ }^{1}$ On a fait ces calculs en regroupant les arbres encore vivants à 15 ans, selon l'étage qu'ils occupaient à 10 ans.

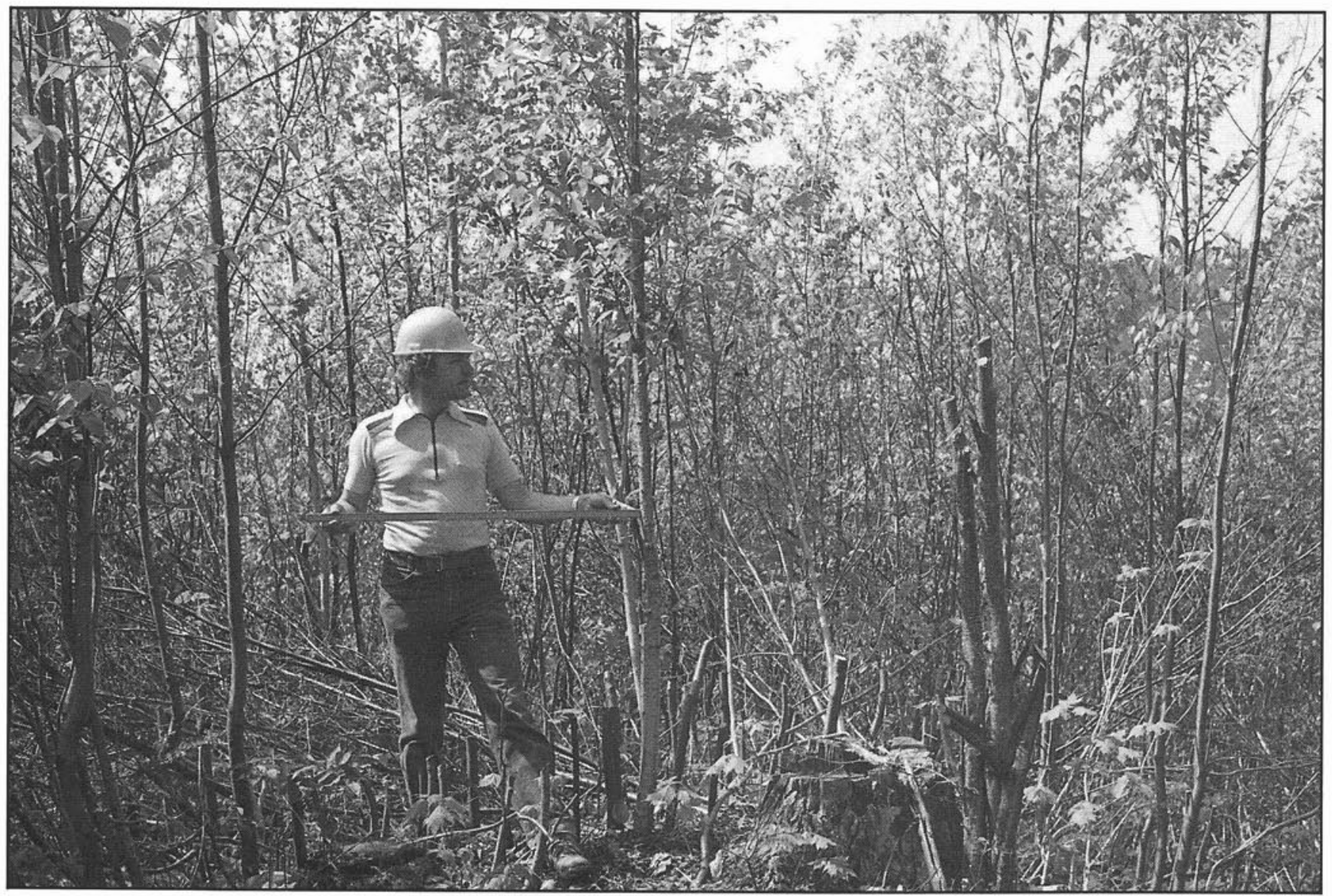

Figure 3. Bouleau jaune de 10 ans au moment du traitement $R_{15}$.

a provoqué des augmentations dans les concentrations en azote $(\mathrm{N})$ des feuilles de l'érable à sucre et du bouleau jaune mais n'a pas eu d'effets marqués sur leur croissance; le dégagement des arbres d'avenir s'avère le traitement le plus efficace pour stimuler la croissance de l'érable à sucre et du bouleau jaune. D'autre part, Penningsfeld (1964) estime que l'érable et le bouleau jaune réagissent particulièrement bien au potassium $(\mathrm{K})$.

Les résultats basés sur l'accroissement en hauteur étaient prévisibles et montrent que celui-ci n'a pas été affecté par 
Tableau 7. Relation entre l'élagage naturel et l'intensité du dégagement chez les bouleaux jaunes dominants et codominants 15 ans après coupe.

\begin{tabular}{|c|c|c|c|c|c|c|c|}
\hline \multirow[b]{2}{*}{ Traitement } & \multicolumn{7}{|c|}{ Présence de la plus grosse branche le long du tronc dans la première bille } \\
\hline & $\begin{array}{l}\text { Aucune } \\
\text { p. } 100\end{array}$ & $\begin{array}{l}<1 \mathrm{~cm} \\
\text { p. } 100\end{array}$ & $\begin{array}{l}1 \text { à } 1,9 \mathrm{~cm} \\
\text { p. } 100\end{array}$ & $\begin{array}{l}2 \text { à } 2,9 \mathrm{~cm} \\
\text { p. } 100\end{array}$ & $\begin{array}{c}3 \text { à } 3,9 \mathrm{~cm} \\
\text { p. } 100\end{array}$ & $\begin{array}{l}4 \text { à } 8,9 \mathrm{~cm} \\
\text { p. } 100\end{array}$ & $\begin{array}{r}\text { Total } \\
\text { p. } 100 \\
\end{array}$ \\
\hline $\mathrm{R}_{0}$ & 58 & 2 & 28 & 8 & 2 & 2 & 100 \\
\hline $\mathrm{R}_{05}$ & 50 & 1 & 32 & 14 & 2 & 1 & 100 \\
\hline $\mathrm{R}_{10}$ & 43 & 2 & 35 & 14 & 4 & 2 & 100 \\
\hline $\mathrm{R}_{15}$ & 8 & 2 & 54 & 27 & 7 & 2 & 100 \\
\hline
\end{tabular}

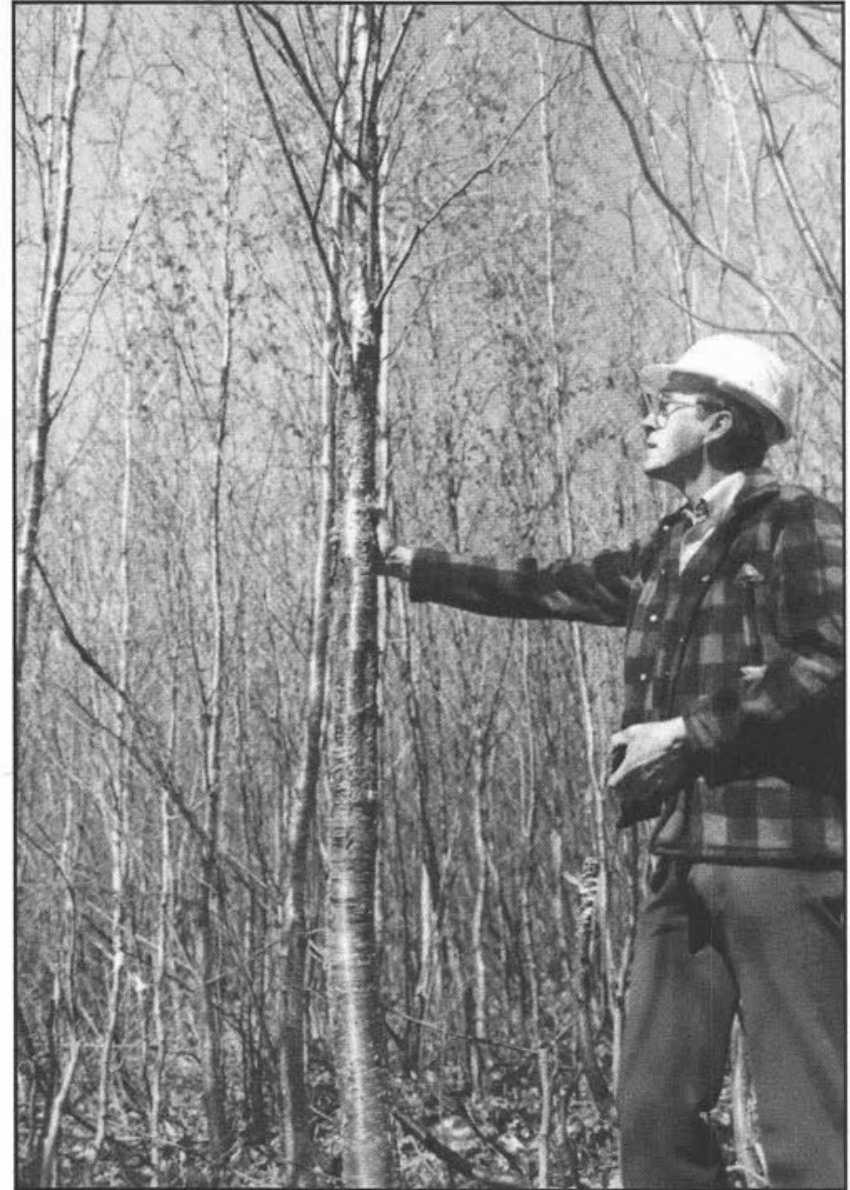

Figure 4. Bouleau jaune de 15 ans, cinq ans après le traitement $\mathrm{R}_{10}$.

les traitements utilisés dans cette étude.

En conclusion, cinq ans après l'éclaircie précommerciale, on obtient les meilleurs résultats d'accroissement en diamètre avec le dégagement fort $\left(\mathrm{R}_{15}\right)$; cependant, l'élagage naturel des gaules est défavorisé pendant cette première période de cinq ans, mais des observations plus récentes nous portent à croire que ceci pourrait se corriger au fur et à mesure que le couvert se referme et que le peuplement s'accroît en hauteur. La fertilisation avec deux combinaisons de $\mathrm{N}$ et trois de N-P-K a eu des effets positifs sur l'accroissement en diamètre dans le cas du dégagement moyen $\left(\mathrm{R}_{10}\right)$ mais quand même trop faibles pour en justifier l'investissement. Le bouleau jaune, espèce semi-tolérante à l'ombre, réagit très bien à l'éclaircie dès l'âge de 10 ans.

\section{Remerciements}

Nous tenons à souligner l'apport de madame Lise Charette, statisticienne, et de monsieur Mario Ménard, ing.f., M.Sc., au traitement des données. Nos remerciements vont aussi à messieurs Jean-Marc Boivin, tech.f., Guy Brousseau, tech.f., Jean-Luc Gauthier, aide-sylv., Jean-Marie Gignac, aide sylv. et Jacques Martineau, tech.f., ainsi qu'au personnel de la Station forestière de Duchesnay dont messieurs Georges Juneau, tech.f., Clément Michaud, tech.f. et Gilles Poliquin, tech.f., pour leur contribution aux travaux sur le terrain, et Fabien Caron, agent d'information, pour avoir participé à la révision du texte final.

\section{Références}

Ellis, R. C. 1979. Response of crop trees of sugar maple, white ash, and black cherry to release and fertilization. Can. J. For. Res. 9: 179-188.

Erdmann, G. G., R. M. Peterson, Jr. et R. M. Godman. 1981. Cleaning yellow birch seedling stands to increase survival, growth, and crown development. Can. J. For. Res. 11: 62-68.

Hannah, P. R. 1985. Response of yellow birch and sugar maple to release and fertilizer. North J. Appl. For. 2: 108-110.

Lamson, N. I. et H. C. Smith. 1987. Precommercial treatments of 15- to 40-year old northern hardwood stands. Dans: Managing northern hardwoods. Proc. Silvicultural Symp. 23-25 June 1986. State Univ. New York. College of Environmental Science and Forestry. Syracuse, New York. R. D. Nylan, Editor. Soc. Am. For. Publ. $N^{\circ}$ 87-03: j160-175.

Noël, C. et R. Rouleau. 1979. Parcelle-échantillon permanente. Normes techniques 1979. Gouv. du Québec, min. de l'Énergie et des Ress., Serv. de l'inv. for. 112 p.

Penningsfeld, 1964. dans Baule H. et C. Fricker, 1969. La fertilisation des arbres forestiers. BLV Verlagsgesellschaft $\mathrm{mbH}$, München.

Robitaille, L. 1971. Inventaire expérimental intensif des compartiments 3 et 4 de la Station forestière de Duchesnay. Gouv. du Québec, min. des Ter. et For., Serv. de la rech. Rapport interne $\mathrm{n}^{\circ} 68,78 \mathrm{p}$.

Rowe, J. S. 1972. Régions forestières du Canada. Ser. Can. For. Publ. 1300F, $172 \mathrm{p}$.

Thibault, M. 1985. Les régions écologiques du Québec méridional. Deuxième approximation. Carte couleurs. Échelle 1: 1250 000. Gouv. du Québec, min. de l'Énergie et des Ress., Serv. de la rech. et Serv. de la carto.

Thibault, M. 1988. Description du milieu forestier du Québec méridional par domaine ou sous-domaine climacique. Gouv. du Québec, min. de l'Énergie et des Ress., Dir. de la rech. et du dév. Rapport interne $n^{\circ} 309,31$ p.

Walsh, L. M. 1971. Instrumental methods for analysis of soils and plant tissue. Soil. Sci. Soc. of America. Madison, Wisconsin, U.S.A., 222 p. 